\title{
Nutzen schaffen für Patienten
}

Richard O. Binswanger

* Aus stilistischen Gründen
wird die männliche Form
verwendet. Frauen sind verwendet. Frauen sind
ausdrücklich mitgemeint.

1 Berry LL, Seltman KD. Management Lessons from Mayo Clinic. Mc. Graw Hill; 2008.

Korrespondenz:

Dr. med. Richard O. Binswanger Radiologie und Nuklearmedizin FMH

Führungsschule Bodensee

Münsterlingen

Oberer Seeweg 9

$\mathrm{CH}-8597$ Landschlacht

r.binswanger@bluewin.ch

www.fsb-spital.ch
Die Aufgabe einer Gesundheitsorganisation ist das Schaffen von Nutzen für Patienten*. Alle andern Aufgaben haben hinter dieser zurückzutreten oder müssen in ihrem Dienst stehen. Andere Anspruchsgruppen sollen hinter den Patienten anstehen.

Für diese Aussage wurde ich als Moderator eines VLSS-Führungskurses von einem sehr erfahrenen Arzt heftig kritisiert. Meine Verblüffung war total. Ist die Aussage falsch? Ist Nutzen das falsche Wort?

«The needs of patients come first.» So lautet der Leitsatz der Mayo Clinic [1]. Das tönt ganz ähnlich. Needs bedeutet allerdings Bedürfnisse und nicht Nutzen. Bedürfnisse können vom Patienten artikuliert oder vom Arzt erkannt werden. Also wäre ich für das Wort «Bedürfnisse» vielleicht nicht angegriffen worden.

\section{Nutzen für Patienten - schwer zu erfassen?} Was mag den Kollegen so erzürnt haben? Wahrscheinlich fand er, dass der Nutzen schwer oder überhaupt nicht zu definieren sei. Auch wenn diesbezüglich Unklarheiten bestehen, der Nutzen lässt sich erkennen. Die Wiederherstellung der Gesundheit, die Besserung einer Krankheit, die Verminderung von Schmerzen, die Begleitung bei unheilbarer Krankheit, die Prävention, alles ist Nutzen. Wiedererlangte Arbeitsfähigkeit nützt dem Patienten mehr als dem Arbeitgeber und der Gesellschaft. Ich gehe noch weiter: Zeitgerechte Terminvergabe für Abklärung und Behandlung, vertiefte Kommunikation, Umgang mit der Angst, Verständnis für nichtmedizinische Probleme und Sorgen, interdisziplinäre Zusammenarbeit, alles schafft Nutzen für Patienten. Nochmals: Der Nutzen ist bestimmbar!

\section{Stillen von Bedürfnissen}

Was Nutzen am Patienten schafft, stillt auch die Bedürfnisse des Patienten. Aber was, wenn Letztere unangemessen sind? Das hat möglicherweise der Kollege gemeint, der in seiner Tätigkeit viele falsche Ansprüche abwehren musste - auf Arbeitsunfähigkeit, Berentung, nach Gefälligkeitsgutachten (Lügen!), auf den ersten Platz in der Warteschlange. Hier muss der Arzt Gegensteuer geben, auch wenn er beschimpft wird oder sonst Nachteile in Kauf nehmen muss. Und mein Verständnis für die Kollegen im Alltag (nicht im ruhigen Befundraum) ist gross.

\section{Wer bestimmt den Nutzen?}

Ausschliesslich der Patient und sein Arzt. Niemand anders. Nicht die Behörden, nicht der Arbeitgeber, nicht die Familie oder die Sozialhilfe. Die beiden Letzteren sollen zwar mitdenken und -reden, aber nicht entscheiden. Den Nutzen zu definieren ist eine vornehme ärztliche Tätigkeit und ein befriedigende dazu. Diese Erwägungen sind allerdings immer subjektiv, das ist auch richtig so, sie gehören in den Bereich der ärztlichen Kunst. Sie sind ausserdem revisionsbedürftig, wenn sich neue Gesichtspunkte ergeben.

\section{Motivation}

Das Schaffen von Nutzen für Patienten ist unsere Hauptaufgabe. Dazu stehe ich, Sie hoffentlich auch. Auch wenn immer wieder versucht wird, uns etwas anderes weiszumachen. Auch wenn andere Aufgaben uns von der Hauptsache ablenken. Es ist unsere intrinsische Motivation. Schaffen und erhalten wir alle Voraussetzungen dafür. Dazu gehört auch die Stärkung und Bewahrung unserer Stellung in der Gesellschaft sowie die Sicherung einer angemessenen Abgeltung.

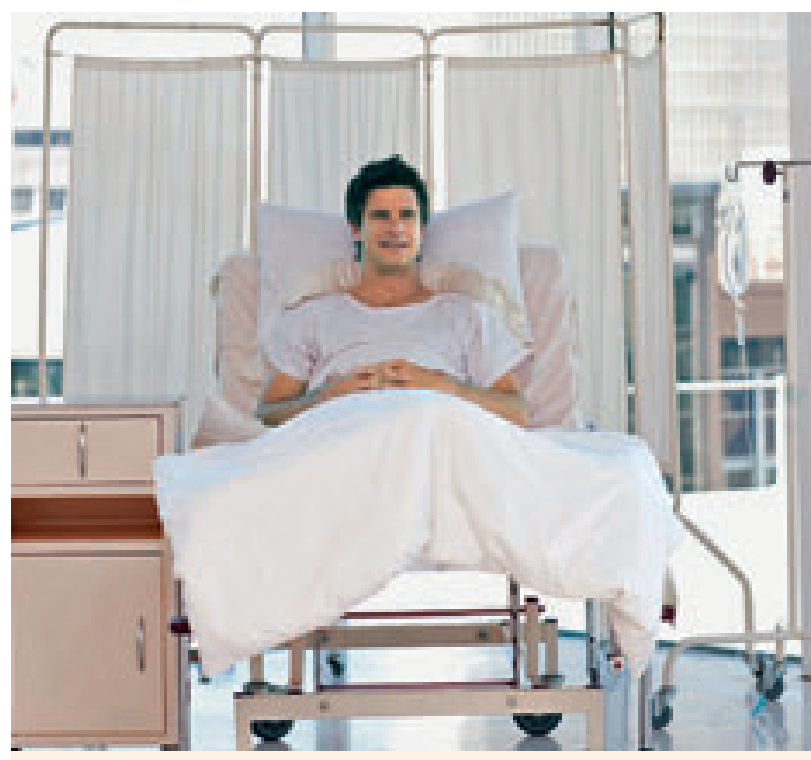

Ist es falsch zu sagen, dass eine Gesundheitsorganisation in erster Linie dem Nutzen des Patienten dienen soll? 